\title{
Entre demonios y elucubraciones: análisis del argumento de Peirce contra el nominalismo'
}

\section{Between demons and elucubrations: analysis of Peirce's argument against the nominalism}

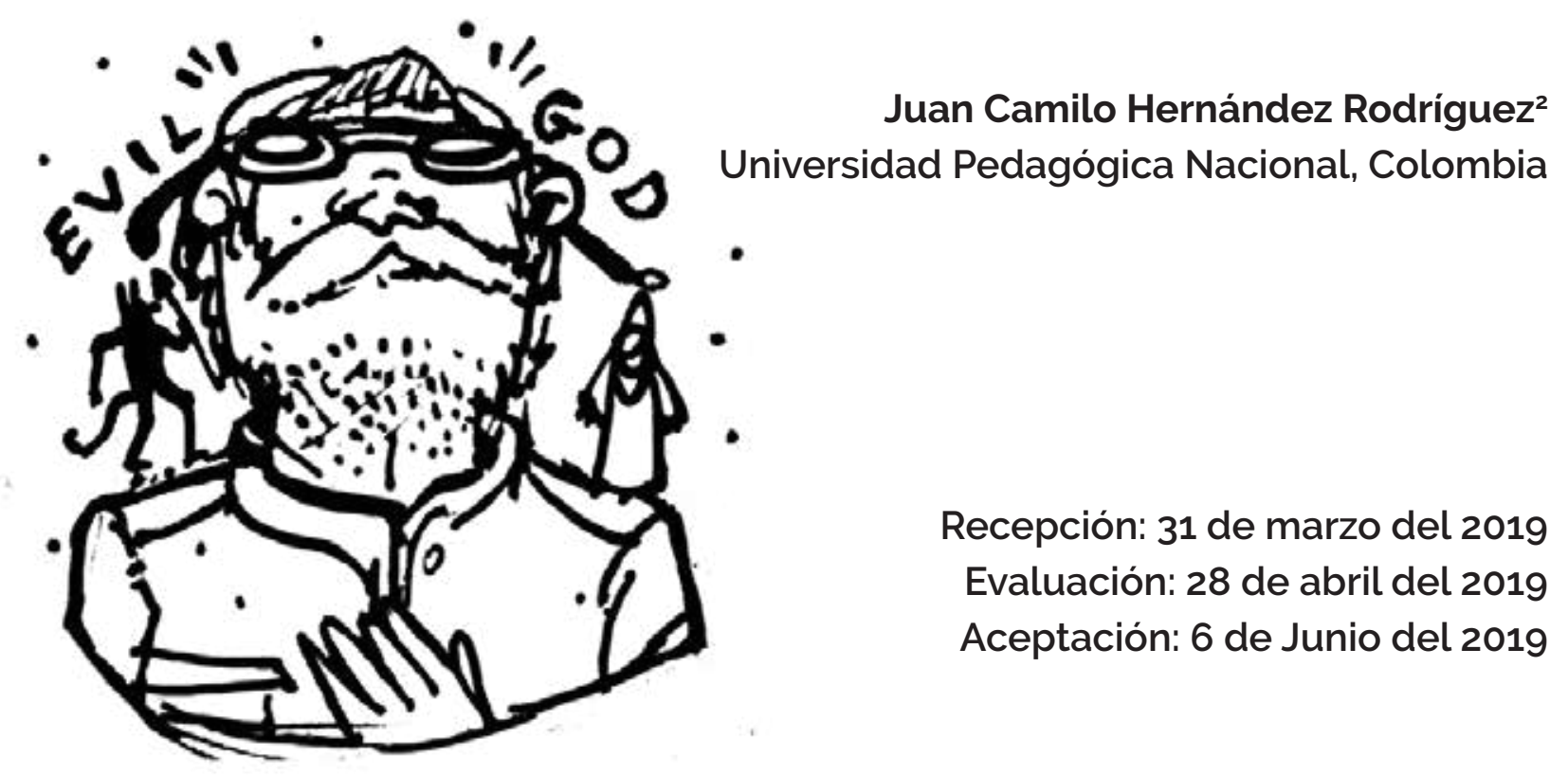

1 Artículo de reflexión derivado de la investigación: « ¿La realidad posee o carece de fundamentos? Análisis y debate entre los conceptos (meta) metafísicos de 'fundamento' (ov̉oía/ ground) y 'origen condicionado' (प्रतीत्यसमुत्पाद [pratītyasamutpāda])».

2 Licenciado en Filosofía de Universidad Pedagógica Nacional. E-mail: If_jchernandezr540@ pedagogica.edu.co 


\title{
Resumen:
}

En este texto, elaboraré un análisis del argumento que Peirce elabora contra el nominalismo en sus New Elements of Mathematics. Para cumplir tal cometido, realizaré un breve recorrido histórico del problema, con el fin de situar la propuesta de Peirce y evaluar su novedad. Luego, expondré su argumento en el marco de su teoria de la primeridad, segundidad y terceridad a nivel metafísico, semántico y lógico. Finalmente, a partir de elementos de la metaontología analítica, evaluaré si el argumento que él ofrece es suficiente para solventar el problema ya mencionado o si, más bien, el debate entre nominalistas y realistas sobre la existencia de los universales es puramente verbal y, por lo tanto, la discusión en la que se enmarca Peirce resulta infértil. Se concluirá que Peirce sí logra superar la discusión, solo que para ello es necesaria una precisión sobre los significados de los conceptos de 'existencia' y 'realidad'.

Palabras clave: concepto, ontología, metafísica, idealismo, materialismo.

\begin{abstract}
:
In this work, I made an analysis of Peirce's argument against nominalism in his article New elements of Mathematics. To fulfill such a goal, I will do a brief historical summary of the problem, trying to locate Peirce proposal and to evaluate its novelty. Then, I will expose his argument in his framework of the theory of primerity, seconarity, and thirdarity in a metaphysical, semantical and logical level. Finally, from these elements of the analytical meta-ontology, I will evaluate if either his argument is enough to solve the already mentioned problem or, rather, the debate between nominalists and realists concerning existence of the universals is only verbal and, therefore, the discussion of Peirce is unfertile. I will conclude that Peirce did surpasses the discussion, but to achieve this he needs precision about the meaning of the concepts of "existence" and "reality".
\end{abstract}

Keywords: concept, ontology, metaphysics, idealism, materialism. 


\section{Parmis des démons et des élucubrations : analyse de l'argument de Peirce contre le nominalisme}

\section{Résumé:}

Dans ce texte, je ferai une analyse de l'argument fait par Pierce, utilisé contre le nominalisme dans ses New Elements of Mathematics. Pour y arriver, j'effectuerai un brief parcouru historique du problème, afin de situer la proposition de Pierce et d'évaluer sa nouveauté. Après cela, j'exposerai son argument dans le cadre de sa théorie Firstness, secondness and thirdness, au niveau métaphysique, sémantique et logique. Finalement, à partir des éléments de la méta-ontologie analytique, j'évaluerai si l'argument proposé par lui est suffisant pour résoudre le problème antérieurement mentionné, ou contrairement, le débat parmis les nominalistes et les réalistes sur l'existence des universels est purement théorique, et donc, la discussion dans laquelle se développe Peirce ne fonctionne pas. À la fin, on pourra conclure que Peirce est capable de surmonter la discussion, en tenant compte qu'il faut une précision sur les significations des concepts de « Existence et Réalité ».

Mots-clés: concept, ontologie, métaphysique, idéalisme, matérialisme. 


\section{Entre demônios e elucubrações: análises do argumento de Peirce contra o nominalismo}

\section{Resumo:}

Neste texto, elaborarei uma análise do argumento que Peirce elabora contra o nominalismonolivroNewElementsofMathematics.Paracumprirtaoconteúdo, realizarei um breve percurso histórico do problema, com a finalidade de situar a proposta de Peirce e avaliar sua novidade. Logo, expondrei seu argumento no marco de sua teoria da primeridade, segundidade e tercereidade no nível metafisico, semântico e logico. Finalmente, partindo desses elementos da metaontologia analítica, avaliarei o argumento para solventar o problema já mencionado ou também, no debate entre nominalistas e realistas sobre a existência dos universais é puramente verbal e, por tanto, a discussão na qual a concepção de Peirce resulta infértil. Conclui-se que Peirce logra ultrapassar a discussão, sobre a necessidade de precisar sobre os significados dos conceitos de "existência" e "realidade".

Palavras-chave: conceito, ontologia, metafisica, idealismo, materialismo. 


\section{Introducción}

Tomemos tres entes cualesquiera; por ejemplo: Sócrates, Platón y Aristóteles. Intuitivamente, diriamos que ellos existen en cuanto tal y no como ideas o producciones de nuestra mente. La razón de ello es que podemos experimentar y tener claras representaciones sobre estos individuos a partir de las cuales podemos predicar con certeza sus cualidades: de Sócrates, que era feo; de Platón, que tenía una espalda ancha; de Aristóteles, que provenía de Estagira. No obstante, no parece ser igual cuando hablamos de términos generales como 'hombre' o 'la humanidad'. Este problema filosófico ha sido denominado el problema de los universales.

Este problema parece ser tan antiguo como la metafísica misma y sobre él se han desarrollado posturas tanto diversas como complejas que, sin embargo, se clasifican en dos: nominalismo (que niega la existencia de los universales) y realismo (que afirma dicha existencia). A pesar de ello, dicho debate sigue siendo muy vigente y teorias cada vez más elaboradas se siguen desarrollando con el fin de solventar el problema (Swoyer, 2008; Dorr, 2008). De todas maneras, el debate se sigue extendiendo tanto y entrando en circularidades que, a nivel metametafísico, ha llevado a autores como Carnap (2005) y Russell (2003b) a creer que este es un pseudoproblema filosófico o, cuando menos, que resulta inocua o puramente verbal la discusión entre nominalistas y realistas (aunque no lo sean sus teorias por separado) (Hirsch, 2002). Estas dos últimas posturas se denominan deflacionismo metaontológico.

En todo caso, hay una propuesta que parece ser novedosa en este debate: la del filósofo Charles Sanders Peirce (1839-1914). En este trabajo, me propongo demostrar que la postura de Peirce en el debate sobre los universales es un conceptualismo que, con la adecuada corrección de términos, logra superar tanto al nominalismo como al realismo absolutos. Teniendo en cuenta la importancia de este debate en la metafísica y en la filosofía en general, considero que la elucidación de esta teoría en el contexto del debate a nivel ontológico y metaontológico tiene gran importancia en lo que artificialmente podriamos denominar 'teorias filosóficas de la realidad'. Para defender esta tesis, aplicaré un método histórico analítico: primero, explicaré de forma general en qué consiste el problema metafísico sobre los universales y 
cuáles son las principales posturas al respecto; segundo, reconstruiré el argumento de Peirce contra el nominalismo y mostraré la diferencia que hay en su teoría entre 'existencia' y 'realidad'. Finalmente, evaluaré si los argumentos de Peirce logran superar los obstáculos mencionados en mi tesis desde tres aspectos metaontológicos: el compromiso ontológico, la variación cuantificacional y un posible criterio pragmaticista de valuación a nivel metaontológico.

\section{Antecedentes: el problema de los universales y sus diversas posturas}

Como puede observarse en el texto de Peirce (1976 [NEM]) y en varios análisis criticos sobre el problema (Ferrater Mora, 1975, t. 2, p. 849, voz 'universales'; Klima, 2017; Beuchot, 1981), el debate entre nominalistas y realistas es fundamentalmente medieval. La razón de ello estriba en que, a pesar de que en la Antigüedad ya existían teorías realistas ( $v$. gr.: Platón y Aristóteles) sobre el tipo de entidad que son los universales, el nominalismo no surgió en Occidente sino hasta la alta Edad Media en voz de Roscelino de Compèigne y de Guillermo de Ockham². Luego, el debate entre realistas y nominalistas acerca de los universales se consolida de forma fuerte y completa hasta la alta Edad Media.

Siendo muy concisos, el problema es el siguiente: ¿es legítimo hablar de un todo (un término universal) en la naturaleza o solo es una ficción de nuestra mente? Retomemos el ejemplo: es un hecho aceptado que existen los seres humanos $x, y$ y $z$; la experiencia sensorial nos da cuenta de ello. Empero, de lo que no nos da cuenta es del género 'ser humano', el cual parece no ser más que la suma de todos los $x, y$ y $z$ que reconocemos con cualidades comunes. Por un lado, quienes aceptan esta última postura -al decir que "'hombre' y 'hombres' significan lo mismo" (de Ockham, 1994, I, c. 44/OPh, 139)- se denominan nominalistas, pues para ellos los universales son solo nombres (convenciones sociales, ficciones mentales o lingüisticas) que inventamos para comprender el mundo de una forma más o menos coherente.

1 Quizás, una teoría protonominalista sea el escepticismo de Sexto Empírico (1996 [PH]), puesto que esta escuela niega la posibilidad de conocer algo más allá de las experiencias propias (I, 18). Sin embargo, siendo consecuentes, un escéptico tampoco se comprometería con un nominalismo, puesto que el nominalista asume dogmáticamente la existencia de individuos y para el escéptico eso todavía no estaría demostrado. 
Esto puede sernos útil, pero elhecho de que ordenemos conceptualmente elmundo no implica que este tenga intrinsecamente este orden o ley natural. En este sentido, los universales son post rem (posteriores a las cosas). El nominalismo suele identificarse con el conceptualismo (teoría en la que se asume que los universales son conceptos) y con el terminismo (teoria en la que se sostiene que esos términos no refieren a entidades en el mundo) (Ferrater Mora, 1975, t. 1, p. 322, voz 'conceptualismo'; t. 2, p. 799, voz 'terminismo'). Filósofos empiristas como Berkeley (1996, int., § 10), Quine (1964) y Goodman (1972) suelen defender esta idea con cierta firmeza.

Por otro lado, tenemos al realista extremo, que sostiene que los términos universales como 'ser humano', 'substancia' o los números son entes que, además de los particulares, también tienen entidad propia y son superiores a las entidades concretas individuales. Ejemplo de ello es la teoría de las ideas de Platón (2011 [Parm.], 135a-d; 2013 [Soph.], 257b-c; Platón, 2014 [Tim.], 27c-28c), en la cual se sostiene que las formas universales (las ideas) son paradigmas que son tenidos en cuenta por el Demiurgo para crear la realidad empírica o región de las apariencias. Luego, los universales son más fundamentales, primarios o substanciales que las cosas concretas; es por esto que estas últimas (las cosas individuales) solo son estudiables en virtud de los primeros (los universales o ideas) (Platón, 2010 [Phaed.], 76e). Dado que son más fundamentales, su existencia es independiente de las entidades materiales. En este sentido, los universales se entienden como ante rem (antes de las cosas). Este realismo ha sido asumido también por Leibniz (1982), Frege (2016), Russell y Whitehead (1903) y Wittgenstein (2009, 6.2-6.22), etc.

Por último, el realista moderado acepta la existencia de entidades con cualidades universales, aunque no las separa o considera independientes de los entes particulares. Más bien, considera que esos principios, leyes, esencias o cualidades universales están en las substancias particulares y es gracias a esto que podemos abstraer de las substancias materiales la esencia de estas y elaborar conceptos. Autores como Aristóteles (2014 [Met.], Z, cc. 2 y ss.), Avicena (1980, tr. V, A195-206, 1-56), santo Tomás (1951 [ST], I, q. 15, a. 1, sol.; q. 16, a. 1, sol.) y Escoto (1960 [Ord.]) han desarrollado teorías más eclécticas que las otras dos ya mencionadas². En este

2 En India, la escuela nyāya - casi contemporánea a la filosofía aristotélica-, también mantuvo una teoría realista moderada. Dice Gotama (1913 [NS]): "nosotros respondemos que no solo el género significa una palabra (nombre), porque la manifestación del género depende de la forma y de la individualidad" (II, 2, § 67). 
orden de ideas, se reconocen los universales ante rem (antes de las cosas), in re (como esencia substancial) y post rem (en nuestro entendimiento).

Sin embargo, Aristóteles (2014 [Met.]) niega tajantemente la existencia ante rem (, 1086b12-1087a25) por considerar que los universales -y las formas en generalsiempre están ligadas a un substrato material (2011b [Phys.], I, 190b29-35). Tomás y Avicena si lo afirman, por reconocer al intelecto de Dios como principio de la creatio ex nihilo. Dado que en el realismo moderado se reconocen ciertos principios o leyes esenciales, los conceptos (universales post rem) si refieren a las cosas (a diferencia del terminismo), aunque se reconocen que son de naturalezas distintas (gnoseológica y ontológica). Esta tesis también fue defendida por Descartes (1989, I, §§55-58) y Peirce (1976 [NEM]). Una clasificación más rigurosa la podemos encontrar en el Diccionario de Ferrater, la cual puede evidenciarse en el siguiente gráfico (véase esquema 1):

Esquema 1. Clasificación de las tesis metafísicas sobre la existencia de los universales.

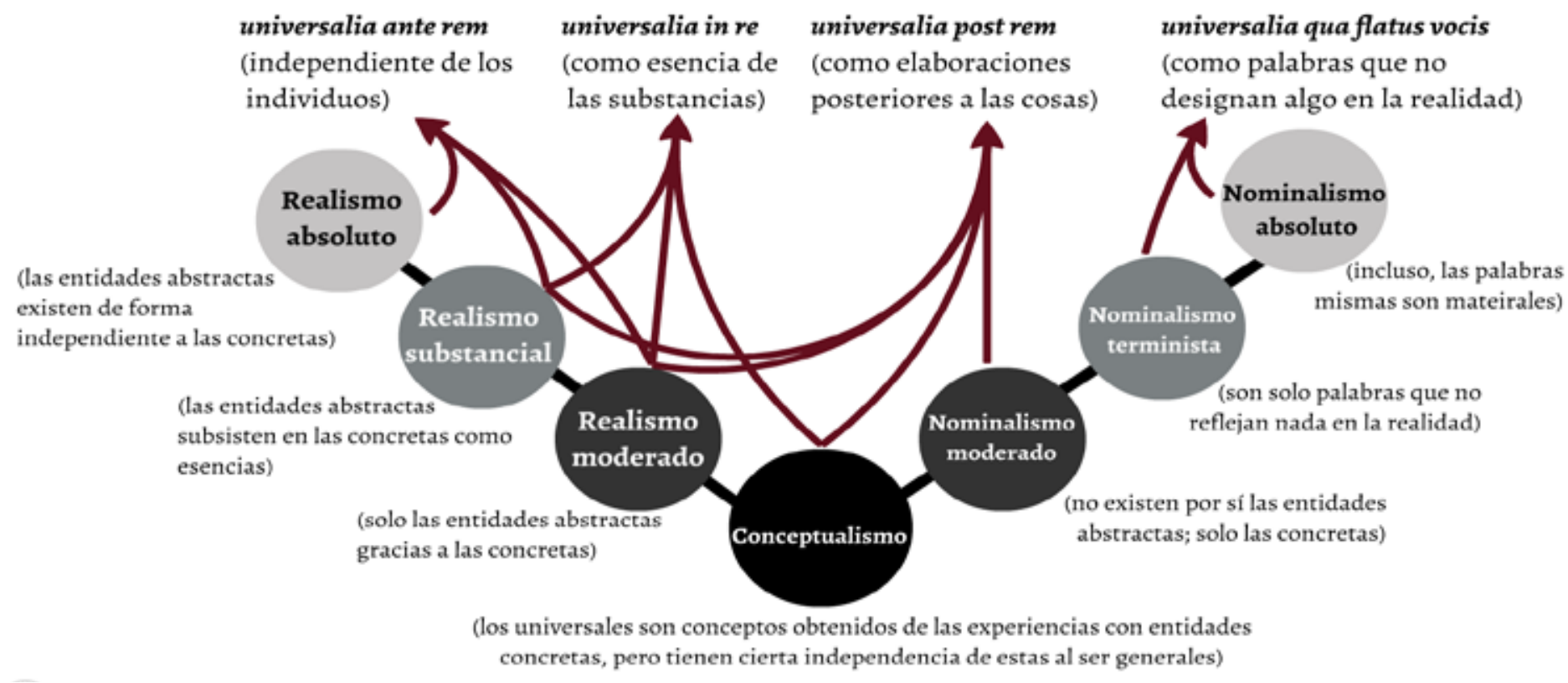

Fuente: elaboración propia, adaptado y basado en la clasificación de Ferrater Mora (1975, t. 2, p. 851).

Ahora bien, la dificultad que presenta el realismo absoluto es que presupone la existencia independiente () de los universales respecto a los particulares, pero no logra demostrarlos sin caer en peticiones de principio. En el caso del nominalismo 
absoluto, sucede lo mismo que en el realismo absoluto, aunque de forma negativa: presupone que no existen los universales o entidades abstractas, pero no demuestra su inexistencia (falacia ad ignorantiam). Por último, las teorías intermedias -como el conceptualismo y el nominalismo y realismo moderados- tienen el inconveniente de tener que explicar cómo se derivan las entidades abstractas de las concretas y viceversa. En el caso de los filósofos medievales, se asume cierto ocasionalismo teísta; en el aristotélico, por intervención de cierto intelecto agente (del cual poco se explica) (Aristóteles, 2011a [DA], III, 7, 431b2-15). Intentar la conexión entre estos tipos de entidades será uno de los retos de Peirce.

\section{La intervención de Peirce: conceptualismo o "realismo científico"}

La posición de Charles Sanders Peirce es curiosa por varias razones. Como él mismo lo indica, inicialmente optó por un nominalismo, influenciado por el empirismo inglés (Peirce, 1994 [CP], 1.149); pero tiempo después asumió un realismo que, basándonos en elesquema anterior, se identifica más con el conceptualismo. Debido a la influencia de Escoto y de la escolástica en general (Beuchot, 1996), dicho distanciamiento o "giro" fue tan radical que posteriormente llegó a denominar al nominalismo como "endemoniado" (Foster, 2011, pp. 1-12).

Peirce arremeterá contra el nominalismo por las siguientes razones: 1. El nominalismo restringe indebidamente el poder de la razón; 2. Habla de los conceptos como ficciones o estratagemas lingüísticas útiles (flatus vocis), pero transitorias; 3. Ve el pensamiento como completamente sometido a leyes deterministas ${ }^{1}$; 4. Socava notablemente la posibilidad de las ciencias normativas; 5. Reduce la realidad a la existencia y no reconoce otras modalidades de ser más que las explícitas (De Tienne, 2016, p. 33). No obstante, ¿cómo logra criticarlo Peirce? Eso lo veremos a continuación; empero, antes de ver su respuesta, es preciso mencionar algunos aspectos generales de toda su propuesta.

\section{Aspectos generales de Peirce}

Tres aspectos generales es preciso tener en cuenta, antes de analizar el argumento de Peirce contra el nominalismo:

1 Este aspecto se debe, según Foster (2011), a que el nominalismo contemporáneo, para la época de Peirce, asumió cierto mecanicismo (pp. 205 y ss.). 
Primero. La teoria lógica del pragmaticismo se funda en tres tipos de inferencia: deducción, inducción y abducción o retroducción. En el primer tipo, se deducen de axiomas verdades necesarias al aplicárselas a casos especificos; en el segundo, se deducen de los casos particulares una probable conclusión; $y$, en el tercero, se conciben a partir de los efectos prácticos de cierto estado de hechos sus hipotéticas causas (Peirce, 1992 [EP1], p. 188). Mientras en el primer tipo de inferencia la conclusión es necesaria y en el segundo es probable, en el tercer tipo es razonable o concebible.

Segundo. Es esta la máxima del pragmaticismo: $x$ es verdadero en virtud de los concebibles efectos prácticos que puede generar en mí (o en varios) (1992 [EP1], p. 132). Tanto el tipo de inferencia como el criterio de verdad descansan en el $\pi \mu$ : es gracias a la hipótesis o insight que podemos hablar de la adquisición de nuevos conocimientos, puesto que, de no haber hipótesis que verificar por la experimentación y que formalizar como ley, no habría necesidad de investigar algo en absoluto. Además, como lo explica Haack (1997), en la propuesta de Peirce se intenta conciliar el criterio empírico (correspondentista o fundacionalista) con el coherentista de la verdad a partir de un elemento común: la praxis (p. 291).

Tercero. La lógica estudia el signo. Hay tres formas lógicas: término, proposición e inferencia (Peirce, 1994 [CP] 1.369-378). Dichos signos son explicados a partir de los principios de primeridad, segundidad y terceridad (' $x$ ', ' $x, y$ ' $y$ ' $x, y, z$ '; o bien: ' $(P \rightarrow Q) \rightarrow R$ ') (1982-2010 [W2]: pp. 103-104); estos son explicables en virtud del tipo de relación que implica (monádica, diádica, triadica) y el tipo de sintaxis en el que se refleja (una sintaxis tricotómica) (Peirce, 1992 [EP1], c. 20).

En el campo semántico (semiótico), el signo obtiene su significado por otra relación tríadica: objeto, signo e interpretante. El primero viene siendo una condición que solo logra develarse por medio de un segundo factor (el signo, bien sea un índice, ícono o símbolo) a un tercero: el interpretante (1994 [CP], 1.300-301; 1.293; 1.303; 1.326-329). Carece de sentido hablar de una teoria del significado sin alguno de estos tres elementos: un objeto e interpretante sin signo (¿cómo se comunicarian entre sí? ¿Por medio de qué?); un signo e interpretante sin objeto (¿sería signo e interpretante de qué cosa?); o, peor, un objeto sin signo e interpretante. ¿De qué estariamos hablando? Respondería Peirce, del $\mu$ kantiano (2011 [KrV], A307), lo cual 
es un absurdo. A partir de estos tres conceptos semánticos, podemos ya vislumbrar por qué para Peirce las teorias nominalista o realista extrema carecen de sentido: porque un nominalismo reduciría el significado al objeto y signo, lo cual trivializa el sentido profundo que este (el significado) involucra. El realismo extremo, por lo demás, exige aceptar realidades nouménicas, innatas o ante rem, lo cual raya con un misticismo poco sano.

\section{Los argumentos contra el nominalismo}

Ahora bien, elucidemos un poco cómo se aplican las categorías de primeridad, segundidad y terceridad en el campo de la metafísica:

La primeridad indica el ser en si de las cosas, previo a cualquier dotación de sentido. Es simple e indeterminada por la mente humana; luego, no puede ser conocida per se. En On a New List of Categories - que es una revisión de las categorias de Aristóteles (1974 [Cat.]) y, principalmente, de Kant (2011 [KrV], A50/B74A64/B88)- Peirce la reconoce como la forma primitiva y más fundamental de la substancia, en el sentido griego de oủoí (1992 [EP1], p. 6).

La segundidad es un proceso de transformación o modificación de ser de un sujeto; empero, su ser no es un ser para nosotros (en una relación tipo objeto-sujeto), sino que es el modo de ser de dos sujetos, una reacción ciega entre dos sujetos (ejemplo: $x$ mueve a $y$ o $y$ recibe un golpe de $x$ ). Es ciega porque todavía no hay un sujeto, un quién, interpretante de ese hecho o interacción entre dos elementos ${ }^{1}$.

En la terceridad, la relación es más clara, dado que la interacción entre esos dos primeros elementos modifica o afecta a un tercero (Peirce, 1976 [NEM], pp. 332-333). En el caso de la triada objeto-signo-interpretante, la manifestación que ese objeto hace por medio del signo a un interpretante es un ejemplo de la terceridad. Otro ejemplo de ello, para retomar los casos anteriores, sería el de la ley (1992 [EP1], pp. 312-333; 1998 [EP2], pp. 67-74)².

1 Según Peirce (1992 [EP1]), inicialmente percibimos la cualidad en un objeto ( $v$. gr:: la estufa negra); sin embargo, para comprender dicha cualidad es necesario apelar a cierto Background o ámbito universal (formal) en el que se comprende la idea universal de 'negrura', gracias a una relación apofántica con sus conceptos negativos respectivos; es decir, " $x$, al ser negro, es 'no blanco', 'no gris', 'no verde', etc" (p. 7).

2 Peirce funda su metafisica y epistemología desde la teoría trascendental de Kant (Haack, 2007).

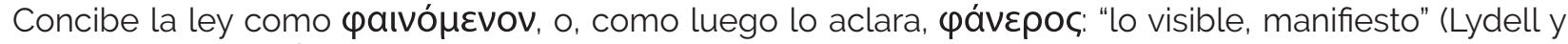

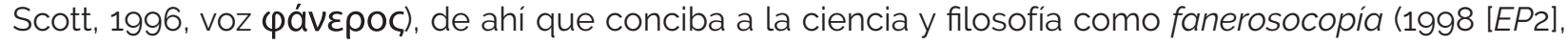
pp. 145 y ss.; cfr. Ballabio, 2016, pp. 19-50). Peirce reconoce dos tipos de representaciones: interna e

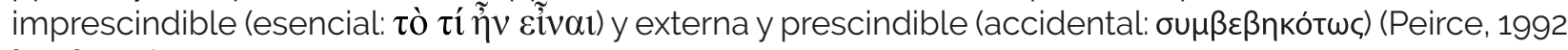
[EP1], p. 7). 
Cuando entra en juego el interpretante es lícito hablar de leyes, pues estas pueden entenderse como cierta regularidad que el interpretante encuentra en la naturaleza. Para usar los términos categoriales: la elevación de la primeridad y segundidad en términos de terceridad (que reúne a las dos anteriores). En la terceridad, la universalidad se ha obtenido por medio de la sintesis entre los dos elementos anteriores; $y$, por ello, el nominalismo ya se ha superado. Empero, esa universalidad no es independiente (como afirman los realistas extremos), sino que siempre es relativa a un quién. Luego, las leyes naturales, los universales y todo principio, concepto o elemento abstracto siempre son dependientes del sujeto que se manifiesta y de los elementos primarios (primeridad [el objeto en sl] y segundidad [el signo]) del que emerge. Claro está que esa dependencia no quita en sentido alguno el hecho de que sea universal.

La primeridad es atractiva para los realistas extremos, puesto que son ellos quienes más se interesan por entender las cosas en si mismas, independientemente de cualquier relación o mediación. La segundidad lo es para los nominalistas, pues estos solo aceptan relaciones (mecánicas y deterministas) ciegas entre dos objetos, pero no logran superar dichas relaciones para ver allí la universalidad, que es propia de la terceridad. En la terceridad, el pragmaticista supera ambas limitaciones y comprende que la universalidad parte de estas dos primeras categorias, pero le rebasa al conseguir alcanzar las leyes mismas de la naturaleza. Dice Peirce (1976 [NEM]):

Finalmente, está la mente geométrica, que está bastante dispuesta a que los demás arrebaten el poder y la gloria siempre que él pueda ser obediente a esa gran vitalidad del mundo que está sacando un cosmos de ideas, que es el fin hacia el cual todos, las fuerzas y todos los sentimientos en el mundo, están tendiendo. Estas son las mentes a las que ofrezco mis tres categorias que contienen algo valioso para su propósito (pp. 333-334).

Ahora bien, una vez explicadas de manera sucinta las categorías de Peirce, queda claro cómo refuta el nominalismo. ¿Cómo lo objeta? Con la lógica. La lógica, dice él, "[...] es la más abstracta y simple de todas las ciencias positivas, y su teoría correcta es bastante indispensable para cualquier metafísica verdadera" (p. 334). Si deducimos lógicamente la terceridad, podremos ofrecer un argumento que ningún 
filósofo serio rechazaría. En este orden de ideas, Peirce retoma la tradicional tarea que Aristóteles (1974 [Cat]; 2014 [Met.]) y los nyāyas de la India (Gotama, 1913 [NS]) trazaron: de la lógica, deducir los principios de la metafísica.

El argumento de Peirce contra el nominalismo puede resumirse de la siguiente manera: Principio 1: no es posible que algo sea una cosa y no lo sea a la vez en el mismo sentido (Aristóteles, 2014 [Met.], , 1005b19-20). Principio 2: Para que se diga que algo tiene una identidad definida, debe existir necesariamente algo distinto de ello que pertenezca a un género común (Aristóteles, 1982 [Top.], 103a7-40). Def. 1: si algo pertenece a un conjunto, pero el conjunto no pertenece a sí mismo, entonces dicho conjunto es normal (Russell, 2016, pp. 575-576).

1. Los nominalistas sostienen la idea de que solo existen los elementos simples (en adelante, los llamaré mónadas para simplificar) y sus relaciones, pero que esto no constituye la existencia de un compuesto o universal. 2. Tomemos todos los elementos simples como una multitud infinita $-v$. gr.: los números naturales- $y$ llamemos a esto el conjunto 'A'. 3. Empero, para que la identidad de los elementos del conjunto sea definida, el conjunto ' $A$ ' debe pertenecer a un conjunto con una iteración mayor: ' $B$ ' (los números racionales). 4. Luego, hay un conjunto de iteración mayor que contiene a ' $A$ '. 5 . Ergo, debe haber un conjunto no contenido en ' $A$ ' que, no obstante, contiene a 'A': 'B'. 6 . Si 'B' contiene a 'A', entonces hay compuestos (porque 'B' es un compuesto al contener a 'A'). Por conclusión (.:), los universales existen (7) y los nominalistas se contradicen (8) (Peirce, 1976 [NEM], p. 344) (véase la formalización en la tabla 1). 
Tabla 1. Formalización del argumento de Peirce contra el nominalismo.

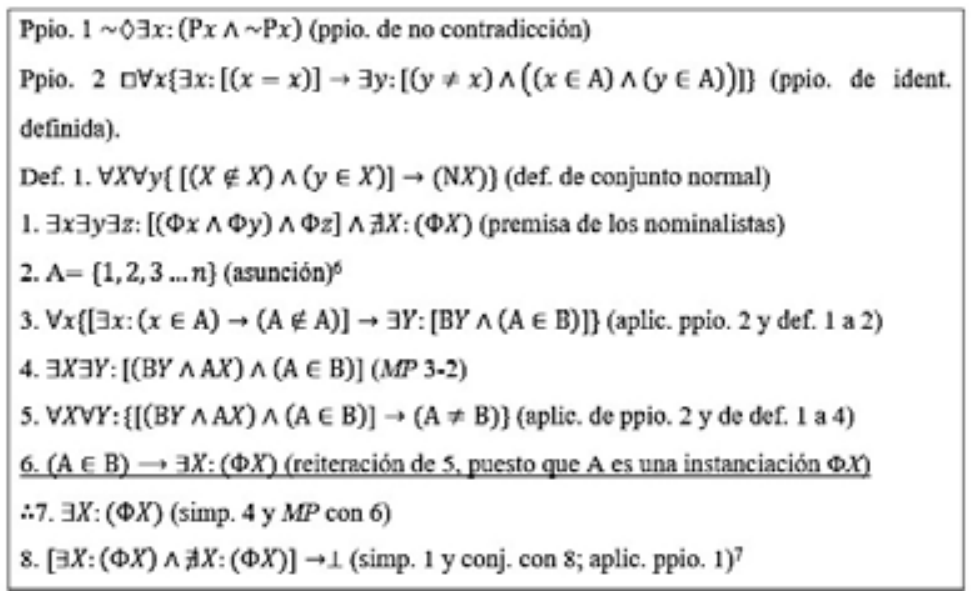

Fuente: elaboración propia, basado en el de Peirce (1976 [NEM], p. 344).

\section{Ahora bien, Peirce ofrece una explicación más cercana al lector no tan familiarizado con las cuestiones matemáticas, a partir de cierto "experimento mentalo psicológico". Miremos los siguientes anillos ilustrados en las figuras por Peirce (véase gráfico 1):}

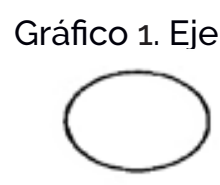

Fig. 2

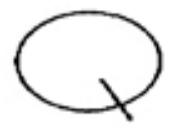

Fig. 3

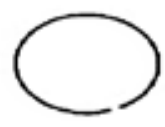

Fig. 4

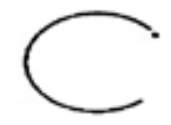

Fig. 5

Fuente: Peirce (1976 [NEM], p. 342)

1 Sea $\mathrm{A}$ el conjunto de los números racionales y $\mathrm{B}$ el de los números reales, como ejemplo de $\Phi X$. En el argumento de Peirce, se denominan denumerales a una multitud de números continuos discretos que, según él, se relacionan generativamente. Dado que hay una multitud denumeral, es necesario reconocer una abnumeral (los números irracionales) que, si queremos reconocer con una identidad definida, deben pertenecer a una multitud mayor: la de los números reales.

2 Sean: 'NX: $X$ es un conjunto normal; ' $\Phi X$ : los $\varphi$ 's; es decir, un predicado o conjunto cualquiera (metavariable); 'A': conjunto de números naturales (es decir, ); y 'B': conjunto de los números racionales (o sea, ). Léase: "Ppio 1: No es posible que algo sea una cosa y no la sea (a la vez y en el mismo sentido). Ppio 2: Para todo $x$, si existe un $x$ tal que es idéntico a si mismo, entonces necesariamente debe existir un $y$ tal que sea distinto de $x$; además de que ambos deben pertenecer a un conjunto superior (A). Def. 1: Para todo conjunto $X$, si ese conjunto no pertenece a si mismo, $y$, además, un $y$ pertenece $X X$, entonces $X$ es un conjunto normal. 1. Existen $x, y$ y $z$ que son $\Phi$, pero no existe $\Phi \times$ (la cualidad universal de $\Phi$ o la " $\Phi$ eidad"). 2. Asúmase el conjunto de los números naturales. 3. Para todo $x$, si existe un $x$ tal que pertenezca a los números naturales, entonces el conjunto de los números naturales no se contiene a sí mismo; luego, existe un conjunto $Y$ tal que es racional y el conjunto de los números naturales pertenece al de los racionales. 4. Existe un conjunto $X$ tal que es natural y uno $Y$ tal que son racional y el conjunto de los números naturales pertenece al de los racionales. 5. Si existe un conjunto $X$ tal que es natural y uno $Y$ tal que son racional, y el conjunto de los números naturales pertenece al de los racionales, entonces los números racionales y los naturales son distintos. 6. Si los números naturales pertenecen a los racionales, entonces tenemos al menos un universal (los números racionales). 7. Existen los universales. 8. Si existen los universales y no existen los universales, entonces hay contradicción (por parte de los nominalistas". 
Según un nominalismo moderado, se afirma que los universales dependen necesariamente de sus partes individuales, de tal modo que el todo se destruye, una vez disueltas las partes. No obstante, si seccionamos un circulo (fig. 2) por medio de una línea (fig. 3), ha de quedar cierto espacio o vacio entre los puntos (fig. 4). Si siguiésemos a raja tabla al nominalismo mecanicista que critica Peirce (que solo reconoce la primeridad y segundidad), al no estar conectados los puntos de forma continua, no habría ese todo (el círculo). A pesar de esto, podemos seguir percibiendo el círculo completo. Esta unidad persiste, aunque la segmentación o el vacío sea todavía mayor (fig. 5). Otro ejemplo de ello lo podemos encontrar en la siguiente figura:

El anterior experimento mental nos sugiere que, tal y como lo propuso Aristóteles, es por lo particular (lo más cognoscible para nosotros) que se alcanza a conocer lo universal (lo más cognoscible en sí mismo); pero, una vez conocido lo universal, a pesar de que lo particular cambie, dicho universal tiene cierta independencia de los cambios particulares; de ahí que le llamemos ley.

Con este último ejemplo puede elucidarse un poco mejor la postura de Peirce frente al problema de los universales: es cierto que los universales se obtienen por medio de los particulares (nihil est in intelecto quid non prium est in sensu); no obstante, tampoco es adecuado decir que el universal no es más que la suma de particulares (de ahí su parcial independencia respecto a estos). Contrario a la interpretación de Beuchot (1996), quien afirma que Peirce es realista casi extremo (p. 1166), el realismo de Peirce es conceptualista (ecléctico), solo que con una importante salvedad que veremos posteriormente.

\section{Análisis metaontológico: ¿se soluciona el problema?}

Luego de haber comprendido los antecedentes del debate y el argumento de Peirce, es preciso que ahora evaluemos si dicho argumento soluciona o no la disputa entre nominalistas y realistas, tendiendo la balanza hacia el realismo. Para hacer esto, evaluaré el problema desde algunos aspectos que son claves en el análisis metaontológico: el problema de la variación cuantificacional y el compromiso ontológico (Quine, 1964), y la inconmensurabilidad de los marcos lingüisticos 
(Carnap, 2005), junto con la posibilidad de ofrecer un criterio para preferir una teoría ontológica por encima de otra (Tahko, 2015, cc. 1-4). En medio de estos dos aspectos, realizaré una precisión a la solución de Peirce.

\section{¿Variación cuantificacional?: mal planteamiento del problema}

Es bien conocida, en el campo de la lógica y la metafísica, la posición de Quine (1964) respecto al compromiso ontológico: cuando utilizamos el cuantificador existencial () nos comprometemos con las entidades sobre las cuales predicamos. Este compromiso no es algo nuevo: las lógicas más antiguas, como la de Aristóteles (1974 [Cat.]) en Occidente y la de Gotama en India (1913 [NS]), asumen este compromiso que, desde el análisis de Quine, toda lógica clásica debe asumir. Este compromiso ha suscitado problemas sobre el tipo de existencia de 'el actual Rey de Francia', 'Pegaso' o 'el círculo redondo' (de nuevo, el problema de los universales). Una opción de solución a este problema es, como lo hizo Russell (1905), apelando a descripciones definidas y reconociendo varios usos del concepto 'existencia' (2003a, pp. 77 y ss.), lo que hace recordar el" $\pi$ " ("lo que es se dice de muchas maneras") (Aristóteles, 2014, [Met.] , 1003a20). Del mismo modo, "existir se dice de muchas maneras" y habría varios sentidos o usos del cuantificador existencial: uno, por ejemplo, para los conjuntos y números; otro, para los entes como tal (Van Inwagen, 2009).

Ahora bien, en este punto, la posición de Peirce es interesante: ciertamente, élasume el compromiso ontológico del lenguaje, solo que hace una salvedad importantísima: que 'realidad' y 'existencia' son conceptos distintos. Dice él: "realidad y existencia son dos cosas distintas" (Peirce, 1994 [CP], 6.349). De hecho, aclara que "realidad significa cierta clase de independencia de nuestro pensamiento, mientras que existencia significa interacción con el entorno [environment]" (5.503). En este sentido, más que haber una variación cuantificacional, el asunto radica en un mal planteamiento del problema. No es que "existencia" sea un concepto multivoco, cosa que niega, quizás, por su influencia de Escoto (Beuchot, 1996). Más bien, de esos dos "sentidos" que Russell le atribuye al concepto de 'existencia', uno de ellos se debe al concepto de 'realidad'.

Para Peirce, la realidad es algo primitivo, fundamental: es primeridad. La existencia, por otra parte, acontece en la terceridad, cuando sintéticamente mente-mundolenguaje se confieren sentido mutuamente en su interrelación (Nubiola, 1998). De ahí que la pregunta clásica, sobre si los universales son entidades reales o no, le parezca inocua. La razón estriba en que está mal planteado el problema por una anfibología 
(confusión en los significados de los conceptos). Dicho problema no se ha podido resolver. Dice él:

¿Qué es la realidad? Tal vez no haya tal cosa en absoluto. Como he insistido repetidamente, no es más que una retroducción, una hipótesis de trabajo que intentamos; nuestra única esperanza desesperada de saber algo. Nuevamente, puede ser -y pareceria muy audaz esperar algo mejor-que la hipótesis de la realidad, aunque responde bastante bien, no se corresponda perfectamente con lo que es. Pero si es que hay alguna realidad, entonces -en la medida en que existe dicha realidad- en lo que consiste esa realidad es el ser de las cosas; algo que se corresponde al proceso de razonamiento: que el mundo vive y se mueve. (Peirce, 1976 [NEM], pp. 344-345).

Así pues, Peirce se compromete con un conceptualismo: "no existen las entidades abstractas en la realidad, sino solo como conceptos de nuestra mente, es decir, como ideas abstractas" (Ferrater Mora, 1975, t. 2, p. 851). Únicamente que, según la aclaración que él hace, habria que agregar: no son reales, pero sí existen. Queda, pues, resuelto el problema sin necesidad de renunciar al compromiso ontológico ni apelar a una variación cuantificacional que multiplique los entes.

\section{Objeción a Peirce: los conceptos están invertidos}

La solución de Peirce parece adecuada en cierto sentido, pero en otro no lo es. 'Realidad' y 'existencia', ciertamente, son conceptos distintos. En ese sentido, sí es verdad que hay anfibología en el debate y, por eso mismo, no ha sido conducido de forma adecuada. Empero, el error de Peirce consiste en que, justamente, la definición de estos dos conceptos está invertida: 'existencia' significa "ser independiente de nuestros pensamientos" y 'realidad' significa "aquella construcción semántica que elaboramos a partir de la existencia (aunque luego logremos rebasarle)".

Tres razones tengo para defender esta tesis. Primero: el término 'existencia' (exsistere), como lo indicó Quine (1964), es significativo; 'ser', en cambio, no lo es. El constructo mismo de la palabra lo indica: "estar por fuera de" (Lewis, Short, Freund, 1960, voz exsisto) ${ }^{1}$. ¿Estar por fuera de qué cosa? De nuestra mente. La distinción entre 'realidad' $y$ 'existencia' podemos encontrarla por primera vez en Escoto (de ahí que Peirce intente distinguirlos de nuevo).

1 Para ahondar más en la definición de 'existencia' (en su sentido cristiano y laico) y su diferencia con 'realidad', véase: Hernández (2018); Hernández y Baracaldo (2018, pp. 328, n. 4; 334, n. 10; pp. 337-338); y Baracaldo y Hernández (2019, p. 14 y ss.). 
En el contexto escotista (Escoto, 1960 [Ord.]), el concepto 'ser' (esse) debe ser unívoco, puesto que ¿cómo podemos decir que algo existe más que otra cosa? Todo existe por igual. Más bien, lo que distingue al ente es su res; es decir, aquello que es el ente (sus formas [cualidades]) (II, dist. 3, pars I, q. 6, n. 15). No obstante, dicha distinción sigue siendo ontológica y no gnoseológica y sigue sin ofrecernos los elementos para resolver el problema ${ }^{1}$. Pero si tenemos en cuenta la teoría epistemológica de Peirce (1992 [EP1]), sabremos que la cualidad siempre atiende a cierto background (p. 7), a un interpretante; conocemos lo negro en virtud del bagaje que tenemos de lo no negro (lo blanco, gris, etc.). En este orden de ideas, al apelar a la teoría misma de Peirce, debería entenderse 'realidad' como conceptual y no como ontológica.

Segundo, porque, como el mismo Peirce (1994 [CP]) indica: "el lenguaje ordinario es garantía suficiente en la materia" (6.361). Naturalmente, así lo es. Nótese que en el uso ordinario del lenguaje decimos "esto es real" como opuesto a lo falso o "verdaderamente esto es asi" para reconocer que efectivamente asi se han dado los hechos. Esta distinción entre 'realidad' y 'existencia' ya la habia hecho Hegel en su famoso aforismo: "Lo que es real [wirklich] es racional, lo que es racional es real [wirklich]" (2010, p. 21; 2017 [Enz.], § 6).

Ahora bien, también es cierto que la gente utiliza el término 'la realidad' queriendo referenciar hechos y confundiendo dicha referenciación con la referencia misma. Lo más probable es que esto se deba a que en la Antigüedad 'ser' tuviera una connotación tanto semántica ("ser verdadero") como ontológica ("existe") (Lydell y Scott, 1996, voz i $\mu$ ). No obstante, actualmente no se concibe a la verdad como una esencia subsistente en las cosas naturales y develable por el intelecto (voces á y ), sino como una construcción humana por medio de los actos y el lenguaje, que es posterior a lo dado. Por ello, de nuevo, el concepto 'realidad' se acerca más a la terceridad que a la primeridad.

Y, tercero, la realidad debe ser conceptual. Precisamente, porque su contenido semántico (ser real: ser verdadero) es lo que necesita la ciencia para tener teorias

1 Es más, como lo evidencia Marquínez Argote (2006), el uso de realidad luego de esta concepción medieval pasó a entenderse como lo real, lo de la realeza, el rey (pp. 151 y ss.); y quería denotar cierta condición de superioridad respecto a algo (en ese caso, de los plebeyos). Valiéndose de esta acepción, también parece que la realidad debe asociarse con la terceridad y no con la primeridad, ya que la terceridad es superior a los dos momentos o estados anteriores, en virtud de su desarrollo y comprensión. 
de leyes o clases naturales. Ciertamente, por esto último es que Susan Haack (2016) interpreta de forma inversa a Peirce, aun cuando él de forma explícita, y desafortunadamente, dice lo contrario:

De acuerdo con esto, la cuestión es: "¿hay universales reales; por ejemplo: leyes y clases reales?" Los hay, pero esto no dice que los universales existan. l... Sin los universales reales no puede haber bases para la explicación, predicción o inducción, y solo lo que podemos hacer es describir hechos y eventos individuales (p. 36).

Esta última observación de Haack es más que atinada: si no reconocemos la realidad de las clases y tipos universales, entonces los discursos científicos no serian más que ficciones o engaños; y es justamente eso lo que pretende sostener el nominalismo. Todo esto se evidencia a partir de las intenciones de Peirce en su empresa investigativa ${ }^{2}$. No obstante, es más que evidente que siempre que Peirce define 'realidad' lo hace como primeridad y cuando se refiere a la terceridad lo hace como 'existencia'. Al menos, así lo indican los textos citados (Peirce, 1998 [EP2], pp. 181-195; 1994 [CP], 8.012).

\section{Conclusión: el criterio pragmático como refutación al nominalismo}

La precisión conceptual que acabamos de realizar tiene que ver con un problema metaontológicoclave:Latraducibilidadentresistemas distintos. Unadelasobjeciones por parte de los filósofos antimetafísicos (Carnap, 2005) es que las teorias metafísicas no pueden discutir entre si porque pertenecen a marcos lingüísticos distintos; en ese orden de ideas, se asume una posición metaontológica deflacionaria (Eklund, 2009). Ahora bien, una solución posible es traducir de un sistema a otro los teoremas que se necesitan evaluar por medio de la lógica de primer orden (Hirsch, 2002 y 2009). En virtud de qué sistema metafísico logre superar los mayores obstáculos, se puede discernir sobre qué teoria metafísica es preferible.

2 Por su parte, Guy Debrock (2001) también cuestiona la inadecuada confusión de Peirce entre lo real y lo existente, entendiendo al primero como simple actualidad, independiente de nosotros y limitada a lo que hay. Un elemento adicional que alli se vislumbra es que la terceridad es siempre pragmaticista, es decir, convoca a nuevos posibles efectos prácticos; por ejemplo: nuevas teorias científicas, modos de vida, etc. En este sentido, 'realidad' entendida como "apropiación semántica" o "construcción de mundo" (que es lo que aqui se sugiere con base en Hegel y Haack) deberia entenderse también en el sentido que aqui sugiero. 
Hayalmenos dos posiblesopciones parasolucionareldeflacionismometaontológico: primero, que ambas teorias metafísicas decidan comprometerse con las mismas definiciones de los conceptos primitivos de los cuales parten-por ejemplo: 'realidad', 'existencia', etc.- y que reconozcan un método verificable común para evaluar los argumentos propios y del adversario (una lógica común); segundo, que se opte por un criterio pragmático (mayor poder explicativo) para preferir una teoria por encima de otra. En el primer caso, las teorias se objetarian; en el segundo, se refutarian.

Respecto a la primera vía de solución al deflacionismo metaontológico, el argumento de Peirce parece ser válido dentro de la lógica clásica. Empero, sigue habiendo un obstáculo: puede darse el caso -como efectivamente lo hay- de que el nominalista apele a que esa identidad de la que se habla en el principio 2 sea solo de dicto y no in re. Es decir, el criterio lógico solo funciona si se presupone -"dogmáticamente", dirian los escépticos- que el mundo se comporta según las leyes de la lógica clásica. Pero esto es lo que no aceptan los nominalistas; luego, sigue persistiendo el problema. El nominalista no acepta ni el criterio de evaluación de los sistemas (la lógica) para resolver la disputa ni mucho menos la distinción entre 'realidad' y 'existencia' que Peirce propone. Por ende, no hay forma de objetar sus teorias o, cuando menos, de tener un debate que vaya más allá de lo "puramente verbal". Quizás, precisamente por eso, a Peirce le parecía que los nominalistas eran "del demonio".

Ahora bien, la segunda vía es más esperanzadora. La teoria de Peirce tiene mayor poder explicativo porque, primero, logra explicar tanto al realismo extremo como al nominalismo a partir de la primeridad y segundidad, respectivamente; segundo, porque puede ofrecer criterios de evaluación interna tanto a nivel lógico (consistencia) como práctico (las posibles aplicaciones en otros campos); tercero, porque no renuncia al compromiso ontológico $-\mathrm{y}$, con ello, al ontologismo- pero tampoco multiplica los entes con pluralismos ontológicos indeseables por razones de economía. En ese sentido, evita los obstáculos que al inicio mencionamos del realismo y el nominalismo clásico: asumir entidades trasmundanas (elucubraciones) de forma dogmática y trivializar el conocimiento científico y filosófico al reducir lenguaje y pensamiento a simples ficciones. Por ello, el conceptualismo de Peirce es preferible al nominalismo ("endemoniado") y al realismo (elucubrado) clásicos. 


\section{Referencias}

Aquinatis, s. T. (1951). [ST] Summa theologiae. Pars I. Madrid: BAC.

Aristóteles. (1974). [Cat.] Categoriae et Liber de interpretatione. Oxford: Oxonii e Typographeo Claredoniano.

Aristóteles. (1982). [Top.] Tópicos. (Trad. M. Candel). Madrid: Gredos.

Aristóteles. (2011a). [DA] Acerca del alma. (Trad. T. Calvo). Madrid: Gredos.

Aristóteles. (2011b). [Phys.] Fisica. (Trad. G. R. de Echandia). Madrid: Gredos.

Aristóteles. (2014). [Met.] Metafísica. (Trad. V. Garcia Yebra). Madrid: Gredos.

Avicena. (1980). Liber de philosophia prima sive scientia divina. Vol. 2. S. Van Riet, ed. Louvain: E. Peeters.

Ballabio, A. (2016). Experiencia y creatividad en C. S. Peirce y M. Merleau-Ponty. Bogotá: Aula de Humanidades.

Baracaldo, C. A. y Hernández Rodríguez, J. C. (2019). De la existencia a la libertad: una lectura metafísica de El ser y la nada, de Jean-Paul Sartre. Saarbrüken, Alemania: Editorial Académica Española.

Berkeley, G. (1995). Tratado sobre los principios del conocimiento humano. (Trad. C. Mellizo Cuadrado). Barcelona: Altaya.

Beuchot, M. (1981). El problema de los universales. México: UNAM.

Beuchot, M. (1996). El realismo escolástico de los universales en Peirce. Anuario filosófico, 29(56), pp. 1159-1172.

Carnap, R. (2005). The Logical Structure of the World and Pseudoproblems in Philosophy. Chicago: Open Court. 
De Tienne, A. (2016). Cómo promover el realismo de Peirce sin ofender al nominalismo. C. Hynes y J. Nubiola (Eds.). Charles S. Peirce. Ciencia, filosofia y verdad (pp. 33-48). San Miguel de Tucumán: La Monteagudo.

Debrock, G. (2001). Las categorias y el problema de lo posible en C. S. Peirce. Anuario filosófico, 34(1), pp. 39-57.

Descartes, R. (1989). Sobre los principios de la filosofía. (Trad. E. López y M. Graña). Madrid: Gredos.

Dorr, C. (2008). There Are No Abstract Entities. T. Sider, J. Hawthorne y D. Zimmerman (Eds.). Contemporary debates in metaphysics (pp. 32-64). Oxford: Blackwell Publishing.

Eklund, M. (2009). Carnap and Ontological Pluralism. D. Chalmers, D. Maley y R. Wasserman (Eds.). Metametaphysics: New Essays to Fundations of Ontology (pp. 130156). New York: Oxford University Press.

Escoto, J. D. (1960). Dios uno y trino. (Trad. F. Alluntis). Madrid: Biblioteca de Autores Cristianos.

Ferrater Mora, J. (1975). Diccionario de filosofía. Dos tomos. Buenos Aires: Sudamericana. Foster, P. (2011). Peirce and the Threat of Nominalism. Cambridge: Cambridge University Press.

Frege, G. (2016). Escritos sobre lógica, semántica y filosofía de las matemáticas. (Trads. X. de Donato, U. Moulines, H. Padilla y C. Pereda). México: UNAM.

Goodman, N. (1972). A World of Individuals. Problems and Projects (pp. 155-172). New York: The Bobbs-Merrill Company.

Gotama. (1913). [NS] The Nyāya Sūtras of Gotama. Allahabad: Sudhīndranātha Vasu. Haack, S. (1997). Evidencia e investigación. Hacia la reconstrucción en epistemología. (Trad. M. A. Martínez García). Barcelona: Tecnos. 
Haack, S. (2007). La legitimidad de la metafísica: El legado de Kant a Peirce, y el de Peirce a la filosofía de nuestros días. Anuario filosófico, 40(2), pp. 471-492.

Haack, S. (2016). The World According to Innocent Realism: The One and the Many, the Real and the Imaginary, the Natural and the Social. J. F. Göhner y E.-M. Jung (Eds.) Susan Haack: Reintegrating Philosophy (pp. 33-55). London: Springer.

Hegel, G. W. F. (2010). Lineas fundamentales de la filosofía del derecho. (Trad. M. Paredes). Madrid: Gredos.

Hegel, G. W. F. (2017). [Enz]. Enciclopedia de las ciencias filosóficas. (Trad. R. Valls Plana). Madrid: Abada.

Hernández Rodríguez, J. C. (2018). Respuestas a las paradojas sobre la omnipotencia de Dios. Pensamiento, 75 (283), 469-485. DOI: 10.14422/pen. v75.i283.y2019.026.

Hernández, J. C. y Baracaldo, C. (2018). Antecedentes metafísicos del concepto ser de Sartre. Universitas philosophica, 35 (71), pp. 323-349. DOI:10.11144/Javeriana. uph35-71.amss.

Hirsch, E. (2002). Quantifier Variance and Realism. Philosophical Issues, 12, pp. 51-73.

Hirsch, E. (2009). Ontology and Alternative Languages. D. Chalmers, D. Maley y R. Wasserman (Eds.). Metametaphysics: New Essays to Fundations of Ontology (pp. 231259). New York: Oxford University Press.

Inwagen van, P. (2009). Being, Existence and Ontological Commitment. D. Chalmers, D. Maley y R. Wasserman (Eds.). Metametaphysics: New Essays to Fundations of Ontology (pp. 472-506). New York: Oxford University Press.

Kant, I. (2011). [KrV]. Crítica de la razón pura. (Trad. M. Caimi). México: FCE.

Klima, G. (2017). The Medieval Problem of Universals. In: Zalta, E. (Ed.). The Stanford Encyclopedia of Philosophy. Revisado el 2 de diciembre de 2019. Retrieved from: https://plato.stanford.edu/archives/win2017/entries/universals-medieval/. 
Leibniz, G. W. (1982). ¿Qué es idea? (1678). (Trad. E. De Olaso). Olaso de, E. (Ed.). Escritos filosóficos (pp. 173-177). Buenos Aires: Charcas.

Lewis, C. T., Short, C., y Freund, W. (1956). Latin Dictionary by Lewis \& Short, Founded on Andrew's Edition of Freund's Latin Dictionary. Oxford: Oxford University Press. Revisado el 2 de diciembre de 2019. Retrieved from: http://www.perseus.tufts.edu/hopper/ text?doc=Perseus\%3Atext\%3A1999.04.0059\%3Aentry\%3Drevisio.

Lydell, H. G., y Scott, R. (1996). Greek-English Lexicon. Oxford: Oxford University Press. Revisado el 2 de diciembre de 2019. Retrieved from:http://wwww.perseus.tufts.edu/ hopper/resolveform?redirect=true.

Marquinez Argote, G. (2006). "Realidad", historia de una palabra desde sus orígenes latinos hasta Zubiri. Cuadernos salamantinos de filosofía, 33, pp. 145-180.

Nubiola, J. (1998). Walker Percy y Charles S. Peirce: abducción y lenguaje. Analogía Filosófica: Revista de Filosofía, Investigación y Difusión, 12(1), pp. 87-96.

Ockham de, G. (1994). Suma de lógica. (Trad. A. Flórez). Bogotá: Norma.

Peirce, C. S. (1976). [NEM] The New Elements of Mathematics. Vols. 1-4. The HagueParis: Mouton Publishers.

Peirce, C. S. (1982-2010). [W] Writings of Charles Sanders Peirce. Vols. 1-4. BloomingtonIndianapolis: University of Massachusetts Press.

Peirce, C. S. (1992). [EP1] The Essential Peirce: Selected Philosophical Writtings. Volume 1 (1867-1893). Bloomington: Indiana University Press.

Peirce, C. S. (1994). [CP] Collected Papers. Vols. 1-8. Cambridge (Mass.): Harvard University Press.

Peirce, C. S. (1998). [EP2] The Essential Peirce: Selected Philosophical Writtings. Vol. 2 (1893-1913). Bloomington: Indiana University Press. 
Platón. (2010). [Phaed.] Fedón. (Trad. C. García Gual). Madrid: Gredos.

Platón. (2011). [Parm.] Parménides. (Trad. Maria I. Santa Cruz). Madrid: Gredos.

Platón. (2013). [Soph.] Sofista. (Trad. A. Tovar). Madrid: CEPC.

Platón. (2014). [Tim.] Timeo. (Trad. F. Lisi). Madrid: Gredos.

Quine, W. V. O. (1964). From a Logical Point of View. Cambridge: Harvard University Press.

Russell, B., y Whitehead, A. N. (1903). The Principles of Mathematics. Cambridge: Cambridge University Press.

Russell, B. (1905). On Denoting. Mind, 14(56), pp. 479-493.

Russell, B. (2003a). The Existential Import of Propositions. S. Mumford (Ed.). Russell on Metaphysics: Selections from the Writings of Bertrand Russell (pp. 77-80). London: Routledge.

Russell, B. (2003b). The Problem of Universals. S. Mumford (Ed.). Russell on Metaphysics: Selections from the Writings of Bertrand Russell (pp. 143-160). London: Routledge.

Russell, B. (2016). Carta de Bertrand Russell a Gottlob Frege [16-6-1902]. Frege G. Escritos sobre lógica, semántica y filosofia de las matemáticas (pp. 575-576). México: UNAM.

Sexto Empírico. (1996). [PH] Esbozos pirrónicos. (Trad. A. Gallego Cao y T. Muñoz Diego). Madrid: Planeta De Agostini.

Swoyer, C. (2008). Abstract Entities. T. Sider, J. Hawthorne y D. Zimmerman (Eds.). Contemporary Debates in Metaphysics (pp. 11-31). Oxford: Blackwell Publishing. 
Tahko, T. E. (2015). An Introduction to Metametaphysics. Cambridge: Cambridge University Press.

Wittgenstein, L. (2009). Tractatus logico-philosophicus. (Trad. J. Muñoz Veiga e I. Reguera Pérez). Madrid: Gredos. 